\section{P054 SLEEP PARAMETERS AND LIGHT EXPOSURE IN A SUB- SAMPLE OF A BRAZILIAN FAMILY-BASED COHORT, THE BAEPENDI HEART STUDY}

${ }^{1}$ Felipe Beijamini*, ${ }^{2}$ Francieli S Ruiz, ${ }^{3}$ Tâmara P Taporoski, ${ }^{4}$ Alexandre C Pereira, ${ }^{5}$ Mario Pedrazzoli, ${ }^{3}$ Kristen L Knutson, ${ }^{2}$ Malcolm von Schantz. ${ }^{1}$ Federal University of Fronteira Sul - UFFS, Realeza, Brazil; ${ }^{2}$ Faculty of Health and Medical Sciences, University of Surrey, Guildford, UK; ${ }^{3}$ Feinberg School of Medicine, Northwestern University, Chicago, USA; Incor, University of São Paulo School of Medicine, São Paulo, Brazil; ${ }^{5}$ School of Arts, Science, and Humanities, University of São Paulo, São Paulo, Brazil

\subsection{6/bmjresp-2019-bssconf.54}

Introduction Sleep is influenced by a multitude of factors, including biological, environmental, as well as sociocultural factors. Evaluating sleep in diverse environments is crucial to develop further understanding of its biological role on health.

Methods Here, we report analysis of a subsample of the Baependi Heart Study. Participants $(n=217$ [82 male]) underwent actigraphy recordings for at least six consecutive days. Activity and light exposure were recorded using 1-minute epochs and visually scored. Means of bedtime, wake up time, time in bed, sleep duration and sleep efficiency were calculated. Comparisons between sexes were performed using Student's $t$ test, and Pearson correlations between sleep, activity and light exposure also were calculated.

Results Comparisons between sexes yielded significant differences for wake up time $(\mathrm{t}(218)=-2.76 ; \mathrm{p}=0.006)$ with women waking up later than men $(07: 30 \pm 01: 46$ vs $06: 54 \pm 01: 05)$, time in bed $(\mathrm{t}(218)=-3.01 ; \mathrm{p}=0.003)$ and sleep duration $(\mathrm{t}$ $(218)=-2.60 ; \mathrm{p}=0.010)$, with longer time in bed $(07: 55$ $\pm 00: 50$ vs $07: 31 \pm 01: 03)$ and sleep duration $(07: 25 \pm 00: 56$ vs 07:02 $\pm 01: 11)$ for women. Total light exposure during the 16 hours of highest exposure (M16lu) was lower in women than men $(\mathrm{t}(218)=4.75 ; \mathrm{p}<0.001$. Greater amount of activity during the most active 10 -hour continuous period was associated with earlier bedtime $(\mathrm{r}=-0.186 ; \mathrm{p}=0.006)$ and earlier wake up time $(\mathrm{r}=-0.185 ; \mathrm{p}=0.006)$. Greater light exposure (M16lu) was correlated with earlier waking up time $(r=-0.206$; $\mathrm{p}=0.002)$, shorter time in bed $(\mathrm{r}=-0.185 ; \mathrm{p}=0.006)$ and shorter sleep duration $(\mathrm{r}=-0.135 ; \mathrm{p}=0.045)$.

Discussion Our finding support the hypothesis that light exposure during daytime is an important factor driving different sleep patterns and timing. This is consistent with the differences we observed between men and women.

\section{P055 \\ COMPARISON OF WATCHPAT TO LIMITED AND FULL POLYSOMNOGRAPHY STUDIES IN ADULTS WITH SUSPECTED SLEEP-BREATHING DISORDERS}

Alain Fildes*, Adrian Kendrick. University Hospitals, Bristol, Bristol Royal Infirmary, Bristol, UK

\subsection{6/bmjresp-2019-bssconf.55}

Background WatchPAT is a portable diagnostic device for studies of suspected Obstructive Sleep Apnoea (OSA) in adults. It is worn as a pulse oximeter and uses Peripheral Arterial Tone (PAT), which reflects sympathetic nervous system activation. PAT allows for complex signal analysis providing detailed information on sympathetic nervous system changes associated with Sleep Disordered Breathing (SDB) events and specific 'signatures' of sleep stages.

Methods Patients were admitted on clinical grounds for overnight sleep studies - either for full (FP: EEG, Respiratory, leg movements and ECG) or limited (LP: no EEG) polysomnography. Patients verbally consented to use the WatchPat device. Simultaneous recordings were obtained from WatchPat and SOMNOscreen PSG (S-Med, UK). This study had internal Trust approval. Studies were manually scored for $\mathrm{SpO}_{2}$ mean, $\mathrm{SpO}_{2}>4 \%, \mathrm{SpO}_{2}$ time $<90 \%$, AHI, Sleep Onset Latency (SOL), Rem Onset Latency (ROL), REM\% and Deep Sleep/ N3(\%), and compared using Bland-Altman analysis. Data are given mean $\pm \mathrm{SD}$.

Results 39 studies were completed of which 13 were FP. The results are summarized in the table 1. For AHI, 25/39 (64\%) of the studies were correctly classified by WatchPat as positive (mild/mod/severe) compared to SOMNO, giving a sensitivity of $75 \%$ and specificity of $80 \%$. Of the studies that were mild (SOMNO), 10/17 (59\%) were correctly identified, with 4/17 classified as normal and $3 / 17$ as $\mathrm{mod} / \mathrm{severe}$. Data from the sleep data showed similarities between the data, although the bias for ROL was wide.

Discussion This study has demonstrated that there is clinically acceptable differences between an in-lab study and WatchPAT for $\mathrm{SpO}_{2}$ and AHI and therefore WatchPat could be used as a clinically acceptable screening tool for suspected OSA. In terms of sleep staging, accepting that the sample size is small $(n=13)$, WatchPat should be used with caution where sleep disorders other than OSA are suspected.

Abstract P055 Table 1 Summary of data comparing WatchPat to the in-lab SomnoMedics device. Data for SOL, ROL, REM\% and N3\% are based on $n=13$. Data for $\mathrm{SPO}_{2}$ and $\mathrm{AHI}$ are based on $\mathrm{n}=39$. Data are given as mean $\pm \mathrm{SD}$. For Bland-Altman, data is mean difference between devices \pm bias of X1 SD.

\begin{tabular}{|l|c|c|c|}
\hline Index & WatchPat & SOMNO & Bland-Altman \\
\hline $\mathrm{SpO}_{2}$ mean (\%) & $95.23 \pm 1.06$ & $94.4 \pm 1.31$ & $0.87 \pm 1.0$ \\
\hline $\mathrm{SpO}_{2}>4 \%$ & $4.65 \pm 9.34$ & $5.5 \pm 10.1$ & $-0.85 \pm 3.05$ \\
\hline $\mathrm{AHI}$ (events/hr) & $9.7 \pm 11.9$ & $9.6 \pm 11.6$ & $0.20 \pm 7.3$ \\
\hline $\mathrm{SOL}$ (mins) & $27.8 \pm 11.3$ & $22.6 \pm 16.6$ & $5.3 \pm 14.9$ \\
\hline $\mathrm{ROL}$ (mins) & $129 \pm 69$ & $128 \pm 65$ & $4.4 \pm 28.7$ \\
\hline $\mathrm{REM}(\%)$ & $19.6 \pm 7.5$ & $18.8 \pm 7.1$ & $1.6 \pm 4.6$ \\
\hline $\mathrm{N} 3(\%)$ & $19.1 \pm 8.2$ & $15.3 \pm 10.2$ & $3.9 \pm 6.6$ \\
\hline
\end{tabular}

